International Journal of Modern Physics D

(C) World Scientific Publishing Company

\title{
GLOBAL AND LOCAL EFFECTS OF ROTATION: OBSERVATIONAL ASPECTS
}

\author{
Włodzimierz Godłowski \\ Institute of Physics, Opole University, \\ Oleska48, 45-052 Opole, Poland \\ godlowski@uni.opole.pl \\ Received Day Month Year \\ Revised Day Month Year \\ Communicated by Managing Editor
}

\begin{abstract}
In the paper we discussed the observational aspects of rotation in the Universe on different scales. We show dependence between the angular momentum of the structures and their size. The presented observational situation is that the galaxies, their pairs and compact groups have a non-vanishing angular momentum. In the structures of mass corresponding to groups of galaxies, this feature has not been found, while in the clusters and superclusters alignment of galaxy orientation has been actually found. Also we know that galaxies have net angular momentum due to the fact that we actually measure the rotation curves of galaxies. These facts lead to the conclusion that theories which connect galaxy angular momentum with its surrounding structure are at some extend favored by data. We show that in the light of scenarios of galaxy formations this result could be interpreted as an effect of tidal forces mechanism, but it is also consistent with Li's model, in which galaxies form in the rotating universe. The theoretical and observational aspects of possible global rotation of the Universe were discussed as well.
\end{abstract}

Keywords: galaxy orientation; angular momenta of galaxies; clusters of galaxies; galaxy formation scenarios; dark radiation; Universe rotation.

\section{Introduction}

Investigating occurrences of rotation in the Universe and its possible consequences is one of the major issues in contemporary astronomy. It involves the question of rotation of individual cosmic structures as well as the question of possible rotation of the entire Universe. The rotating objects in the Universe are found on various scales - from subatomic particles to stars and galaxies - so it is reasonable to ask whether the Universe as a whole does rotate too. If there is the global, or just a large-scale, rotation of the Universe, its effects should be able to be detected in an observational way. In this work, the possible rotational effects at various astronomical scales are discussed and observational data that could be possibly related to rotation are presented.

The attempts to observationally confirm that the Universe actually rotates have been ever regarded much skeptically. The first significant evidence for the global 
rotation of the Universe was provided by Birch (1982). Birch considered position angles and polarization of classic bright double radio sources and found that the differences in position angles and their polarization are correlated with their position on the sky. That study was immediately criticized by Phinney and Webster (1983), who accused Birch of invalid application of statistical methods and stated that his data are inadequate for drawing such far-reaching conclusions. In reply to them, Birch (1983) found that the effect observed by him had been present in the original data of Phinney and Webster, while the validity of Birch's statistics was proved by Kendall and Young (1984). In next work by Phinney et al. (1984), the data were reanalyzed using novel statistical methods and taking into account possible errors and observational uncertainties. The authors concluded that the effect indicated by Birch was confirmed by observations, however its nature was not clear. Bietenholz and Kronberg (1984) and Bietenholz (1986) having extended the sample of analyzed objects did not find any evidence to confirm Birch's effect. Another attempt to empirically confirm the rotation of the Universe was undertaken by Nodland and Ralston (1997a), who studied correlations between the direction and distance to galaxies and the angle $\beta$ between the polarization direction and their larger axis and found an effect which they interpreted as rotation of the polarization plane dependent on the distance. The study immediately provoked discussion on the validity of statistical methods used (Carrol and Field 1997, Loredo et al. 1997, Eisenstein and Bunn 1997, Nodland and Ralston 1997b, see also Ralston and Jain 2004 for later review); it was also indicated that the effect had not been confirmed by analysis of new, better observational data (Wardle et al. 1997, see however that Jain and Ralston 1999 obtained opposite conclusion), and that even if Birch's effect were to be regarded as evidence for a large-scale rotation of the Universe, the value obtained by Birch is too large as compared to the anisotropy found in cosmic microwave background radiation (CMBR). Potential difficulties in empirical confirmation of rotation of the Universe were mentioned also in a number of theoretical works. Silk (1970) already drew attention to the fact that at present, otherwise than in the early Universe, any dynamic effects of global rotation should be negligible, and the rotational period must exceed the Hubble time, which is a direct consequence of the small anisotropy of CMBR. Later on, Barrow, Juszkiewicz and Sonoda (1985) obtained strict constraints on the value of rotation scalar by investigating some classes of Bianchi models which involve Friedmann' models as their special cases. They demonstrated that for a flat universe there is a limit of $\omega / H_{0} \sim 2 \times 10^{-5}\left(\omega \sim 1.5 \times 10^{-15} \mathrm{rad} \mathrm{yr}^{-1}\right)$. Recently Pontzen and Challinor (2007), examining effects of CMBR polarization induced by global rotation, demonstrated that they could be used to determine constraints on the amount of global rotation. Su and Chu (2009) obtained a limit of this kind from analyzing the 2nd-order Sachs-Wolfe effect, namely that the angular velocity of shear-free rotation of $\Lambda C D M$ universe is less than $\omega \sim 10^{-9} \mathrm{rad} \mathrm{yr}^{-1}$ at the last scattering surface. This constraint is weaker than that obtained by Barrow, Juszkiewicz and Sonoda 
(1985) for the flat Bianchi models. Chechin (2010) investigated the rotational effects of cosmic vacuum. Considering the global rotation and the induced rotation of elliptical galaxies, he estimated the value of angular velocity of the Universe in his model as $\omega \sim 10^{-19} \mathrm{rad} \mathrm{s}^{-1}\left(3 \times 10^{-11} \mathrm{rad} \mathrm{yr}^{-1}\right)$. In the light of the above results the global rotation of the Universe cannot be regarded as confirmed by observations, thus instead of observational confirmation of rotation of the Universe one should rather talk about rotational limits within a given cosmological model.

The relativistic models with rotation and geodetics in spacetime behaving according to general relativity were investigated already in the first half of 20th century, beginning from the works of Lanczos (1924), Gamow (1946) and Goedel (1949). Ellis and Olive (1983) as well as Gron and Soleng (1987) demonstrated that the global rotation in inflationary models of the Universe - if any - should be small. Demiański and Griszczuk (1972) discussed solutions of the Einstein equations for the case of flat homogeneous anisotropic space filled with expanding and rotating ideal fluid with non-zero shear, addressing primarily the question how rotation influences the behaviour of matter near the initial singularity. The Einstein equations with rotating fluid were also analyzed in a number of studies by Krasiński (Krasiński 1975, 1997, 1998a,b,c, 1999, 2001a,b). Fil'chenkov (2003, 2005) considered a possibility of cosmological origin of rotation of astronomical objects due to a tunneling effect in quantum cosmology. The dynamics of Friedmann-Robertson-Walker (FRW) models with global rotation was discussed in Szydłowski and Godłowski (2005). The role of rotation of objects in the Universe and its significance for astronomical measurements was analyzed in Vishvakarma (2006). Recently Bratek, Jalocha and Kutschera (2007) found a class of explicit solutions with cylindrical symmetry for differentially rotating gas. Discussion of anisotropic cosmological models with magnetic field and rotation can be found in Demiański and Doroshkevich (2007).Jain, Modgil, and Ralston (2007) examined the Type 1a supernova data in order to determine if it shows any signal of large scale anisotropy. The anisotropy was modeled by an extended Gödel metric (Gödel-Obukhow metric), which incorporated expansion along with rotation. Sousa, Pereira and Silva (2008) considered the relation of energy and momentum density in Goedel's universes, while Akarsu and Kilinc (2010) analyzed locally rotationally symmetric (LRS) Bianchi Type I cosmological models are examined in the presence of dynamically anisotropic dark energy and perfect fluid. Iorio (2010) provided a detailed account of influence of large-scale rotation on the Solar System.

The main problem in testing models with non-vanishing rotation is that there are no accepted observables for this purpose to date. It is also worth noting that while there have been established some upper limits for rotation from analysis of CMBR and primeval nucleosynthesis (Goedel 1949, Hawking 1969, Collins and Hawking 1973, Barrow, Juszkiewicz, and Sonoda 1985, Ciufolini and Wheeler 1995, Bunn, Ferreira and Silk 1996, Kogut, Hinshaw and Banday 1997), all these works based on a model involving both shear and rotation, which makes discussing rotation a 
rather complicated task due to intertwined effects of shear and rotation. Thus it is easier to study a Newtonian equivalent of this model, which allows for rotation with no shear.

\section{Cosmological models with dark radiation}

In looking for limits of amount of rotation it is convenient to use Senovilla's formulation of Newtonian cosmology. According to Senovilla, Sopuerta and Szekeres (1998), density and pressure in a Newtonian universe filled with homogeneous fluid will not explicitly depend on spatial variables, while depending on time. It is, however, assumed that fluid velocity depends in a linear manner on spatial variables (Szekeres and Rankin 1977, Senovilla, Sopuerta and Szekeres 1998). In this case, contrary to the general relativity solutions, there are solutions without shear that fulfill the Heckmann-Schucking equations with expansion and rotation. This situation has no equivalent in general relativity, where homogeneous rotating and expanding universes (but non-tilted - the models where vectors of 4 -velocity $u^{\mu}$ are not orthogonal to the surface of homogeneity are called "tilted" ones - Obukhov (2000), Obukhov, Chrobok, and Scherfner 2002)) with ideal fluid have to possess a non-vanishing shear (Ellis 1966, King and Ellis 1973, Raychaudhuri 1979, Collins 1985). In a Newtonian universe one can readily derive observables required for cosmological tests. On the other hand, the Newtonian approximation seems a reasonable one, as it is revealed from the CMBR analysis that the Universe is flat or nearly flat (Spergel et al. 2007), and furthermore, for the flat Bianchi I models, for one, the shear scalar decreases more rapidly (with increasing scale factor) than the rotation scalar in an expanding universe with ideal fluid as the field source (Hawking 1969, Ellis 1973, Rothman and Ellis 1986, Li 1998).

The motion of fluid in a homogeneous Newtonian universe can be described by the expansion scalar $\theta$, rotation tensor $\omega_{a, b}$, and shear tensor $\sigma_{a, b}$. The global rotation of the Universe consists here in homogeneous fluid rotating as a whole (Li 1998). The equation representing Newtonian cosmologies, with expansion and rotation but without shear, which should be regarded as the principal equation of the Newtonian homogeneous cosmology is called the Heckmann-Schucking equation (Heckmann and Schucking 1959, Heckmann 1961). It was Heckmann (1961) who demonstrated that such a Newtonian model can be constructed in a consistent manner.

From observations of distant supernovae of Ia type (Perlmutter et al. 1998, 1999, Riess et al. 1998) as well as measurements of anisotropy of cosmic background (Spergel et al. 2006) it follows that the Universe accelerates its expansion and is of nearly flat geometry. It raises a question if this acceleration is due to rotating fluid and if the results of Ia-type supernovae observations can be explained by the effects of rotation. Distance measurements, such as measuring luminosity distance as a function of redshift $\mathrm{z}$, are sensitive to the spatial geometry of the model and evolution of scale factor. Thus the observed luminosity of a supernova 
(after taking into account extinction, K-term correction, etc.) will depend on the current density of various components of matter and their equations of state. The Heckmann-Schucking equation in Newtonian cosmology plays an analogical role as the Friedmann equation in relativistic cosmology. From our point of view it is essential that in the Newtonian analogy of Friedmann equation the rotation effect produces a negative term scaling as $(1+z)^{4}$, which formally corresponds to a presence of (fictitious) fluid of negative density scaling as radiation and thus called "dark radiation". The presence of such a fluid would be much desirable, since, as it was demonstrated by Ichiki et al. (2002), it could contribute to accounting for the discrepancies between the abundances of helium- 4 and deuterium theoretically predicted in the model of primordial nucleosynthesis (BBN) and the observed ones. A preliminary analysis (Godłowski and Szydłowski 2003b) showed that while the rotation effects underlied the acceleration of the Universe, they alone are not sufficient to explain the observations of Ia-type supernovae, which would require additionally some kind of "dark energy", for instance, the cosmological constant. It should be noted that the constraints on rotation in Godłowski and Szydłowski (2003b), which could be derived from supernovae observations at that time, were quite weak.

The problem of determining limits on rotation of the Universe was addressed in a more detailed way in the next article (Godłowski and Szydłowski 2006). In analyzing observational effects of rotation, the Heckmann-Schucking equation was used as the simplest model in which they are separated from the other effects and thus the influence of rotation can be considered in a relatively easy way. This work took up observational limits to a negative term of $(1+z)^{4}$ type in the Friedmann equation (or its Newtonian equivalent - the Heckmann-Schucking equation). The estimation of amount of rotation from the microwave background radiation (CMBR) and primordial nucleosyntheis (BBN) was made assuming the presence of additional, non-interacting fluid - radiation matter (of density parameter $\Omega_{r, 0}$ ). The constant appearing in the Heckmann-Schucking equation was regarded as a curvature constant, which, as the Newtonian cosmology is flat by definition and its curvature constant is zero, was actually a departure from the Newtonian model.

However, in that work it was noted that a negative term scaling as $(1+z)^{4}$ can be present in the Friedmann equation (or its Newtonian equivalent) for a number of reasons. The main interpretations here, apart from a cosmological model with global rotation, are "dark radiation" in Randall-Syndrum's brane scenario (Randal and Sundrum 1999, Vishwakarma and Sing 2003) and the Casimir effect. Unfortunately, on the base of astronomical observations only, it is not possible to distinguish particular components of such a fluid scaling as radiation, as cosmography tests the Universe's properties through the relation $H(z)$, which does not allow for differentiating components if they scale identically with changing of the scale factor. Thus the analysis yields a sum of density parameters of such fluids instead of constraints for pure rotation. So in the work of Godłowski and Szydłowski (2006) the $\Sigma \Omega_{d r, 0}+\Omega_{r, 0}$ of density parameters of all (real and fictitious) fluids scaling as radiation was esti- 
mated instead. It means that the limit on the net contribution of density parameters from all such fluids, both of positive (as radiation) and negative (as Casimir's effect) energy density. In the analysis, the observations of supernovae (Riess et al. 2004, Astier et al. 2005) as well as those of IIb-type Fanaroff-Riley radio galaxies (Daly and Djorgovski 2004) were used. Combining data from supernovae and radio galaxies to determine values of cosmological parameters was proposed by Daly and Djorgovski (2003), who also noted the fact that for the purpose of investigations of this kind, one had better use coordinate distance (Weinberg 1972) instead of luminosity distance, commonly used in analysing data from supernovae. Coordinate distance, as opposed to luminosity distance does not depend on the value of Hubble constant $H_{0}$, but only on the density parameters appearing in the model of (real or fictitious) fluids. Using the coordinate distance in the work of Godłowski and Szydłowski (2006) had also this advantage that it allowed for making a consistent use of the constraints on cosmological parameters derived from the baryon oscillation peak (Eisenstein et al. 2005) and the shift parameter in CMBR (Wang and Tegmark 2004), which do not depend on the value of $H_{0}$ either.

However, while in the case of Fanaroff-Riley IIb-type radio galaxies Daly and Djorgovski provide coordinate distance for each galaxy, for supernovae both Riess et al. (2004) and Astier et al. (2005) give just the value of luminosity module (apparent luminosity minus absolute luminosity), from which one can readily obtain luminosity distance. Still, in order to calculate coordinate distance out of luminosity distance one needs the value of Hubble constant. In Godłowski and Szydłowski (2006) two samples of supernovae were analyzed, for which luminosity modules had been derived in a somewhat different way. For Astier's sample, they were obtained assuming a priori $H_{0}=70 \mathrm{~km} \mathrm{~s}^{-1} \mathrm{Mpc}^{-1}$ (Astier et al. 2005), and this value was used in calculating coordinate distances for supernovae in this sample, while for Riess's sample the value of Hubble constant should be calculated independently beforehand (in the considered model $H_{0}=64.4 \mathrm{~km} \mathrm{~s}^{-1} \mathrm{Mpc}^{-1}$, or $h=0.644$ was obtained) and then used in further calculations.

Eventually, it was shown that the flat model of the Universe with density parameter $\Omega_{m, 0}=0.3$ is favoured by observations, while the value of $\Omega_{d r, 0}$ parameter is at best estimated as zero, which corresponds to vanishing rotation. Thus the sought-after net negative contribution of fluids scaling as radiation, if any, is small. One can also obtain a limit on the value of density parameter $\Omega_{d r, 0}$, which is $\Omega_{d r, 0}>-0.00035$ at the confidence level 0.95 , but this means that the sum of density parameter $\Omega_{d r, 0}$ and radiation density $\Omega_{r, 0}\left(\Omega_{r, 0} \sim 0.0001\right.$, Vishwakarma and Singh (2003)) is also negative. In that case we would have the bouncing scenario instead of the Big Bang scenario (Molina-Paris and Visser 1999, Tippet and Lake 2004, Szydłowski et al. 2005), where the Universe does not start its evolution from the Bing Bang, but initially contracts to its minimal size (when the squared Hubble constants is zero), and then begins to expand. It should be noted that for the value $\Omega_{d r, 0}=-0.00035$ the Universe would bounce already at the redshift $\mathrm{z}$ of about 1200 , so the primordial nucleosynthesis would not have a chance to occur. 
Since the primordial nucleosynthesis is a well-established aspect of cosmology, it imposes strict constraints on the values of $\Omega_{d r, 0}$. Basing on the estimates of Ichiki et al. (2003) it can be asserted that at the confidence level of 0.95 , the primordial nucleosynthesis permits the values $-7.22 \times 10^{-5}<\Omega_{d r, 0}<0.65 \times 10^{-5}$. So there's a need of determining a stronger limit on the estimated parameter $\Omega_{d r, 0}$. Such a possibility is provided by analysing the first peak in the power spectrum of CMBR.

It should be stressed that there is a discrepancy between the value of matter density parameter $\Omega_{m, 0}$ in Godłowski and Szydłowski (2006), consistent with the value obtained earlier from supernovae observations (Riess et al. 1998, Perlmutter et al. 1999, Riess et al. 2005), and the value obtained by Spergel et al. (2006) from observations of background radiation $\left(\Omega_{m, 0}=0.24\right)$. Furthermore, there is a discrepancy between the values of Hubble constant used in early studies of the Universe's acceleration based on observations of Ia-type supernovae (Perlmutter et al. 1999, Efstathiou et al. 1999, Vishvakarma 2001) and the values $H_{0}$ obtained from the CMBR analysis (Spergiel et al. 2007). It is usually accounted for as an effect of uncertainty in determining the zero point for Cepheids used in calibrating the Hubble constant. For instance, Freedman et al. (2001) suggest an inaccuracy of $\pm 8 \mathrm{~km} / \mathrm{s}$ as an effect of systematic errors. However, other researchers, as Tuner (2010a, 2010b), do not confirm that there are such systematic errors involved. By analyzing CMBR, Spergel et al. (2007) determined that the density parameter $\Omega_{m, 0}=0.128 h^{-2}$. For $\Omega_{m, 0}=0.30$ (Perlmutter et al. 1998, 1999, Riess et al. 1998, $2004,2007)$ the value of the Hubble constant is $H_{0}=65 \mathrm{~km} \mathrm{~s}^{-1} \mathrm{Mpc}^{-1}$, but then for the flat Universe in the classic scenario of dark cold matter with a cosmological constant $(\Lambda C D M)$ there is a discrepancy between the theoretical location of the first peak and the observed one. The proper location of the first peak in this model is obtained for $H_{0}=73 \mathrm{~km} \mathrm{~s}^{-1} \mathrm{Mpc}^{-1}\left(\Omega_{m, 0}=0.24\right)$. In the considered work of Godłowski and Szydłowski (2006) it was shown that the presence of fluid of negative density scaling as radiation permits to avoid these discrepancies, and so we can get a flat model of the Universe with $\Omega_{m, 0}=0.3\left(H_{0}=65 \mathrm{~km} \mathrm{~s}^{-1} \mathrm{Mpc}^{-1}\right)$, consistent with both the Ia-type supernovae and CMBR observations. In this model Godłowski and Szydłowski (2006) obtained at the confidence level 0.95 a strict constraint on the sum of density parameters $\Omega_{d r, 0}$ of negative fluids scaling as radiation: $-1.05 \times 10^{-5}<\Omega_{d r, 0}<-0.5 \times 10^{-5}$. This limit is stronger than that obtained earlier by Ichiki et al. from analysis of nucleosynthesis and CMBR. It should be also stressed that the obtained net value $\Omega_{d r, 0}+\Omega_{r, 0}$ is greater than zero, which means that the Big Bang scenario and not the bouncing one is valid indeed.

\section{Li's model and its predictions}

In $1998 \mathrm{Li} \mathrm{Li}$-Xin proposed a model involving galaxy forming in a rotating universe. The very idea that as a consequence of the conservation of angular momentum in a rotating universe, the galaxies acquire its angular momentum during their formation, was already considered by Gamow (1946), Goedel (1949), and later by 
Colins and Hawking (1973). One of its drawbacks was that it predicted alignment of galaxies' rotational axes, which was not confirmed observationally at that time. $\mathrm{Li}$ (1998) explored the issue of galaxy formation in a rotating universe in a more detailed manner. He analysed the model of homogeneous, rotating and expanding universe, filled with ideal fluid, which additionally complies with the laws of energy and angular momentum conservation. In Li's model, when the fluid's equation of state is $p=0$ (dust), the shear scalar $\sigma^{2}$ decreases with increase of the scale factor as $a^{-6}$ (i.e. $\sigma^{2} \sim a^{-6}$ ), while the rotation scalar $\omega^{2}$ diminishes more slowly $\left(\omega^{2} \sim a^{-4}\right)$. Thus it is quite reasonable to presume that (for a sufficiently large scale factor) that in comparison with rotation, shear is negligible, since it decreases more rapidly than the rotation scalar with increasing the scale factor. The model is based on the Steigman-Turner equation (Steigman and Turner 1983). Interesting comments on this equation and its applicability can be found in Rothman and Ellis (1986).

The relation between the mass of a cosmic structure and its angular momentum is usually expressed by the empirical relation $J \sim M^{5 / 3}$ (Wesson 1979, 1983, Carrasco, Roth and Serrano 1982, Brosche 1986), which origin has been discussed for a long time. One of the first attempts to account it for was made by Muradyan $(1975,1980)$, who tried to explain it in terms of the Ambarzumian's superdense cosmogony. Wesson (1981) pointed out its possible role in the unification of gravitation and particle physics and later (Wesson 1983) argued that it is a consequence of self-similarity of Newtonian problem applied to rotating gravitationally bound systems, while Mackrossan (1987) involved thermodynamical consideration for its explanation. Sistero (1983) introduced the problem of rotational velocity of the Universe into considerations. The same line of thought was taken by Corrasco et al. (1982), who viewed the relation $J \sim M^{5 / 3}$ as a consequence of mechanical equilibrium between the gravitational and rotational energies. Li (1998) indicated that in the model of rotating universe the relation $J \sim M^{5 / 3}$ is a direct consequence of the law of angular momentum conservation. The correlation between the mass of spiral galaxies and their angular momentum was considered in a detailed manner by a number of authors (e.g. Nordsiek 1973, Dai, Liu and Hu 1978, Cerrasco, Roth and Serrano 1982, Abramyan and Sedrakyan 1985). Investigating this correlation, $\mathrm{Li}$ (1998) estimated the Universe's rotation and found the amount close to that obtained earlier by Birch (1982). Heavens and Peacock (1988) show that the relation $J \sim M^{5 / 3}$ is also a prediction of the tidal torque scenario (such attempts were made, among others, also by Catelan and Theuns 1996).

In Godłowski et al. (2003a) it was pointed out that in Li's model the relation between the angular momentum of a cosmic structure and its mass is of a more complicated character than simply $J \sim M^{5 / 3}$ (which is just its approximation for large masses M). Actually the relation is of the form $J=k M^{5 / 3}-l M$, where $\mathrm{k}$ and $l$ are constants dependent on the amount of the Universe's rotation, density of matter, size of a protostructure and the moment of its formation, as well as the parameter $\beta$, determined by the mass distribution within the protostructure. 
Consequently, the relation $J(M)$ has the global minimum $J_{\min }$ for the mass $M_{\min }$. It was also shown that $M_{\min }$ does not depend on the amount of rotation of the Universe, while depending on the size of forming protostructure and the moment of its formation (redshift $z_{f}$ ), as well as on the value of parameter $\beta$ (it is presumed to be close to 1). Dark matter was ignored in the considerations, since it was assumed to be collisionless and non-interacting with the observable matter in any other way than by gravitational force. Thus the mass estimates provided below pertain to the mass of dust structures (and not the total mass including dark matter present in galaxies and clusters of galaxies).

In the discussed work it was also demonstrated that under reasonable assumptions, the structures of certain masses should possess a vanishing angular momentum. One can then check if the results from observations of galaxy structures are consistent with the predictions of Li's model. But one should have in mind numerous difficulties in theoretical estimating of $M_{\min }$. It involves a number of approximations since values of such parameters as mass distribution within the protostructures, their size, and the moment of forming of galaxies and clusters are not known. The latter parameter depends strongly on the accepted scenario of formation of galaxies and their clusters. However assuming some reasonable values of those parameters, one can get some estimate of $M_{\min }$. This theoretical minimum should be then confronted with the relevant observational data.

From the observational point of view of interest is the absolute value of angular momentum $|J|$, since in general the direction of structures is not known, while one can quite readily identify the situation when angular momentum vanishes or is very small. The vanishing angular momentum $\mathrm{J}=0$ is obtained when the structure's mass is $M_{0}=2.15 \times M_{\min }$. We should keep in mind that Li's model remains valid only provided rotation occurs on a sufficiently large scale. Moreover, due to its scaling properties Li's model can be applied to rotating protostructures of various initial size. Thus our considerations concerning angular momenta of galaxy structures will be also valid in the case of large-scale, but not necessarily global, rotation of the Universe. So the model can be of interest even if we reject the hypothesis of global rotation of the Universe.

In Godłowski et al. (2003a) it was attempted to estimate the masses of structures of minimal angular momentum in relation to the size of protostructure. And thus, if we assume that clusters of galaxies arise as a result of collapse protostructure (Sunyaev and Zeldovich 1972, Dorohkevich 1973), then the structures of minimal angular momentum should be of a mass of galaxy clusters. In the case of protostructure of protogalaxy size, its mass should be of an order of globular cluster, while in the case of protosolar cloud it should be comparable to the giant moons of the Solar System. Except for the Moon, the angular momenta of such celestial object are actually smaller than the angular momenta of either planets or asteroids (Wesson 1979). On should note however that formation of the stars inside the galaxy or the formation of a solar system is such complex astrophysical process that extrapolation 
of Li's (1998) and Godłowski (2003a) results to a protosolar cloud is questionable.

It raises a question if there is any possibility to observationally test the predictions of Li's model. Such a possibility is provided by studies of galaxy plane orientation.

\section{Galaxy orientation and galaxy formation scenarios}

Studies of galaxy plane orientation were conducted already in 19th century (Abbe 1875). The methods and results of investigations performed up to Second World War can be found in the article of Danver (1942), today only of historical value. The first postwar work was the cited to this day treatise of Holmberg (1946), who compared the numbers of galaxies seen face-on and edge-on, discussed the observational effects related to optical measurements of size of galaxy axes, and proved that the observed excess of edge-on galaxies is just of observational origin. Typical for the early period after the Second World War are the analyses of Wyatt and Brown (1955) and Brown $(1964,1968)$, devoted to investigating distributions of position angles within the galaxy-rich regions of the sky: Cetus (Wyatt and Brown 1955), Pisces (Brown 1964), and in Hydra, Sextant, Ursa Maior, Virgo and Eridanus (Brown 1968). In his two studies Brown $(1964,1968)$ discovered a departure from isotropy of position angle distribution. Reinhardt (1970) and Reinhardt and Roberts (1972) analysed distributions of position angles of large semiaxes of the galaxies from the Reference Catalogue of Bright Galaxies (de Vaucouleurs 1964) and found a very weak preference of galaxy plane alignment with the equator plane of the Local Supergalaxy. Nilson (1974) investigated the galaxies brighter than $14.5^{\mathrm{m}}$ from his UGC catalogue (Nilson 1973). His results are consistent with those obtained by Reinhardt and Roberts. However either results are compromised by the presence of galaxies not belonging to the Local Supercluster in the analysed sample.

Further important progress in investigations of galaxy plane orientation was made by Hawley and Peebles (1975), who in a detailed manner discussed the method of investigating galaxy orientation through analysing distribution of position angles as well as possible errors and observational effects, in particular the earlier results of Brown, indicating their insufficient certainty due to possible observation errors.

Investigating orientation of galaxy planes in space is of importance, as various scenarios of formation and evolution of cosmic structures predict different distributions of angular momenta of galaxies, i.e. provide different predictions concerning orientation of objects at different levels of structure - in particular clusters and superclusters of galaxies. This provides a method for testing scenarios of galaxy formation. One assumes that normals to the galaxies' planes are their axes of rotation, which seems to be quite reasonable, at least for the spiral galaxies.

In the scenario of hierarchic clustering (Peebles 1969, Doroshkevich 1970, Dekel 1985) large-scale structures in the Universe form "from bottom up", as a consequence of gravitational interactions between galaxies. This means that galaxies form in the first place and only later group into larger clusters. The galaxies' spin angu- 
lar momentum arises as an effect of interaction with their neighbours. In original version of this model the orientation of galaxy rotational axes would be random. One should note however that in the hierarchical clustering theory naturally arises the Tidal Torque scenario. In this scenario galaxies have their angular momentum perpendicular to the surrounding structure due to the coupling between the protogalaxy region and surrounding structure.

On the other hand, the scenario of primordial turbulences (von Weizsacker 1951, Gamow 1952, developed further by Oziernoy 1978 and Efstathiou and Silk 1983) accounts for the spin angular momentum as a remnant of the primordial whirl and predicts that the rotationalaxes of galaxies would be perpendicular to the main plane of a large-scale structure.

Another approach is represented by Zeldovich's pancake model (Sunayev and Zeldovich 1972, Doroshkevich 1973, Shandarin 1974, Doroshkevich, Saar, and Shandarin 1978, Zeldovich 1978), which provides that structures in the Universe form "from up to bottom". In the effect of asymmetrical collapse of a large structure there arises a magnetohydrodynamic shock wave, which causes the structure to fragment as well as imparts galaxies with spin angular momentum. In this scenario the mechanism of gaining angular momentum by galaxies consists in acquiring rotation by a shock wave passing across the protostructure, which gives rise to a coherent, nonrandom orientation of galaxy planes in space. Thus the model predicts the galaxies' rotational axes to be parallel to the main plane of the structure.

It should be stressed that this classic view in which each of the basic scenarios predicts a different alignment of rotational axes of galaxies was later somewhat disturbed. It was shown that in principle in any scenario, including that of hierarchical clustering, can happen a phase of shock wave, which may give rise to alignment of rotational axes. Naturally, the extent of this alignment is differentiated.

Two main methods of investigating alignment of galaxy axes have been proposed to date. The first one, consisting in examining distributions of position angles of galaxies, was presented in detail by Hawley and Peebles (1975). Hawley and Peebles (1975), and subsequently Kindl (1987) discussed detailedly the statistical methods they suggested for probing position angle distributions. Unfortunately, the distributions provide reliable data on galaxy plane orientation only for the galaxies observed edge-on. In consequence, the galaxies seen face-on or nearly face-on have to be excluded from the analysis. Furthermore, in studying nearby structures the galaxies have to be located in the vicinity of the structure's main plane. For instance, when considering galaxies belonging to the Local Supercluster (LSC), we should take into account only galaxies located at small supergalactic latitudes B (Godłowski 1994).

Jaaniste and Saar $(1977,1978)$ improved on a novel method proposed by Oepik (1970), which took into account not just galaxy position angles but also their ellipticities (angles of inclination to the line of sight). In this method, using galaxies of any possible orientation and location on the sky, one calculates two angles. One is the angle between the normal to the galaxy's plane and the main plane of the structure (polar angle) and the other one is the angle between the normal projected onto 
the main plane of the structure and the selected direction on this plane (azimuth angle). For example, in the study of galaxies belonging to the Local Supercluster it can be the line connecting our Galaxy with the centre of the Virgo cluster. After determining those angles, they can be analysed using statistical methods to look for any non-random trends. Unfortunately, in this method for any galaxy there are two possible orientations, corresponding to two possible positions of the normal to the galaxy plane in space. Since usually the sense of galactic angular momentum is not known, there are actually four possible orientations of a galaxy's rotation axis. Flin and Godłowski (1986) improved on the method of Jaaniste and Sarr (1978), correcting the errors present in their original work, and described the method of analysing distribution of vectors normal to the galaxies' planes, basing on the statistical methods proposed by Hawley and Peebles (1975) and adapting them for the purpose of investigating angle distributions in this case. The methods were further developed in the subsequent works by Godłowski $(1993,1994)$. In Jaaniste and Sarr's (1978) method one has to take into consideration both the fact that galaxies are flattened spheroids (Holmberg 1946), with their actual ratios of axes depending on their morphological type (Heidmann, Heidmann, de Vaucouleurs 1971), and the Holmberg effect (Holmberg 1946, 1958, 1975, Fouque and Paturel 1985). The Holmberg effect consists in the fact that micrometric measurements of larger and smaller semiaxes of galaxies depend on ellipticity of the image, which in turn creates a necessity of reducing the micrometric axis ratio $\mathrm{q}$ to the standard photometric system. Fouque and Paturel (1985) derived the formulae for reduction of galaxy sizes obtained from micrometric measurements in various galaxy catalogues to a uniform system of photometric diameters. How the actual ellipticity and the Holmberg effect influence the results of galaxy orientation analysis was discussed in Flin and Godłowski (1986, 1989a) and Godłowski $(1993,1994)$.

The methods of investigating of galaxy orientation have been further scrutinized and improved ever since. A critical analysis of the method of investigating spatial orientation of galaxies using their ellipticity was provided in Godłowski and Ostrowski (1999), where it shown that the process of deprojection of galaxies basing on the catalogue data generates significant systematic errors, which have to be taken into account in analysing alignment of galaxy rotation axes. The effect, confirmed by Godłowski, Baier and MacGillivray (1998), and Baier, Godłowski and MacGillivray (2003), could have led to partly distorting the earlier results concerning galaxy orientations.

Cabanela and Dickey (1999) indicated difficulties in detecting rotational axis alignment for small samples. Analysing a sample of 54 edge-on galaxies with spin vectors well-determined from $\mathrm{HI}$ observations, they demonstrated that even a considerable amount of anisotropy in distribution of galaxy spins within the Perseus cluster, signalled in the work of Cabanel and Aldering (1998), could have well remained undetected.

Several variants of the methods mentioned above were used in investigating 
rotational axis alignment by a number of authors (see e.g. Hu $(1995,1998), \mathrm{Wu}$ $(1997,2006)$ Aryal Saurer (2000)). Modifications of the original methods were also made in order to adapt them to some particular research problems (Lee and Pen 2001, Brown et al. 2002, Noh and Lee 2006a, 2006b, Trujillo, Carretero and Patiri 2006). And thus Lee and Pen (2001) described their method of investigating intrinsic galaxy alignment in respect to the local tidal shear tensor and discussed in detail the method of determining this tensor as well as examining correlation both between the galaxy spins and between the spins and the local tidal shear tensor. In turn, Brown et al. (2002) discussed a method of investigating intrinsic alignment of ellipticities of galaxies based on their ellipticity variance. Noh and Lee (2006a, 2006b) examined alignment of planes of spiral galaxies seen edge-on in respect to the plane of local "pancake". This plane was determined locally for each individual galaxy, defined as a plane passing through the galaxy and its two nearest neighbours. Trujillo, Carretero and Patri (2006) studied the distribution of angles between rotational axes of galaxies and the vector connecting a given galaxy with the centre of a local "void" (thus introducing a vector normal to the local plane of the structure as defined by the ambient matter).

\section{Results of investigations of galaxy orientations in the Local Supercluster and its close neighbourhood.}

The orientation of galaxies in the Local Supercluster and its close neighbourhood was studied by many researchers. Most of the early works on the alignment of rotational axes (Wyatt and Brown 1955, Brown 1964, 1968, Reinhardt 1970, Reinhardt and Roberts 1972, Nilson 1974, Jaaniste and Saar 1978, MacGillivray et al. 1982a,b, MacGilivray and Dodd 1985a,b, Kapranidis and Sullivan 1983) implied that the galaxies are either oriented in a random way or the galaxies' planes are parallel to the structure's main plane. And thus MacGillivray et al. (1982a,b) scrutinizing the distribution of spiral and irregular galaxies belonging to the Local Supercluster found a weak correlation between the galaxy planes and the main plane of LSC. These results confirmed either the theory of hierarchic clustering or the theory of primordial turbulence. At that time just Jaaniste and Saar (1978), using not only position angles of galaxies but also their inclination, claimed that in LSC there is an excess of galaxies with rotational axes aligned in the equatorial plane of LSC.

The question of existing discrepancies between the results of early research on galaxy orientation was discussed in the articles of Flin and Godłowski (1986) and Godłowski $(1993,1994)$. They were caused mainly by contamination of the samples with background objects as well as difficulties with proper interpretation of the obtained results due to using the equatorial coordinate system instead of the supergalactic one. For instance, Kapranidis and Sullivan (1983) analysed the density of positions of galactic poles within regions of $30^{\circ} \times 30^{\circ}$ size for a sample of spiral galaxies in the equatorial coordinate system. The authors claimed that there had been no departure of galactic pole distributions from the expected isotropic 
distribution, as they found an excess of galactic poles in only two of the analysed regions, while it escaped their notice that these very regions $\left(\alpha=195^{\circ}, \delta=15^{\circ}\right.$ and $\left(\alpha=15^{\circ}, \delta=-15^{\circ}\right)$ are in the direction to the centre and anticentre of the Virgo cluster (i.e. the centre of the Local Supercluster) respectively, which means that in fact they discovered that the galaxy planes prefer the orientation perpendicular to the plane of the Local Supercluster.

The question of proper choice of a coordinate system should be kept in mind when analysing orientation of galaxies in clusters and superclusters other than LSC. In such a case the most appropriate one could be the coordinate system connected to the main plane of the analysed structure (Flin 1994, Godłowski 1995). The importance of proper choice of a coordinate system was also stressed by Bukhari and Cram (2003), Wu (2006), and particularly Aryal, Kandel and Saurer (2006), who showed that that the interpretation of the results of probing galaxy orientation in the Abell 3558 cluster - the core of the Shapley Concentration - depends on the choice of coordinate system.

Flin and Godłowski (1986) and Godłowski $(1993,1994)$ demonstrated that the planes of galaxies belonging the Local Supercluster are oriented perpendicularly to the main plane of the LSC equator, while the effect depended on the galaxy's morphological type. This result was later confirmed by the study of Parnovski et al. (1994), which found an excess of galaxy rotational axes directed towards $4-6^{h}, 20-$ $40^{\circ}$. The authors recognized that the observed anisotropy is of global character. This is generally consistent with the earlier results of Fliche and Soriau (1990) concerning the orientation of extended galactic HI envelopes and the "cosmic pole" detected in the analysis of remote quasars $\left(5^{h} 30^{m}, 7^{o}\right)$. However, Flin (1995) upon analysis of these results indicated that the observed anisotropy is consistent with the results of Flin and Godłowski (1986) and is not of global character but specific to LSC.

However these results seem to favour the pancake model of Zeldovich, there are arguments that the situation is much more complicated. Flin and Godłowski (1990) and Godłowski (1993) studied the orientation of galaxies belonging to various substructures of the Local Supercluster, defined basing on the work of Tully (1986) and showed that the orientation is different for different substructures. Godłowski (1994) provides the dependence of orientation on radial velocity of the galaxies under investigation. Furthermore, in Flin and Godłowski (1989a), Kashikawa and Okamura (1992) and in Godłowski (1994) it was shown that the rotational axes of galaxies at low supergalactic latitudes are parallel to the main plane of LSC, while the rotational axes of galaxies at high supergalactic latitudes are perpendicular to that plane. In this case the galaxy rotational axes tend to be directed towards the centre of the Virgo cluster. This effect, confirmed recently by Hu et al. (2006), supports a more complicated hybrid model rather than the simple pancake model. Also the results of Aryal and Saurer (2005a), who found a weak preference of spin directions for the spiral galaxies belonging to the Local Supercluster, do not agree with the predictions of Zeldovich's pancake theory. Aryal and his collaborators in the series of articles (Aryal, Kafle and Saurer 2008, Aryal, Neupane and Saurer 2008, Aryal, 
Paudel and Saurer 2008, Aryal 2010) analysed various samples of spiral galaxies in the Local Supercluster and its neighbourhood (with radial velocities $V<5000 \mathrm{~km} / \mathrm{s}$ ) and found that only the barred spirals manifest alignment of orientation of galactic planes, while the alignment effect depend on their radial velocities.

On the other hand, Lambas indicated that the alignment of galaxy orientation can be of local character. Lambas, Groth and Peebles (1988) compared the position angles of elliptical galaxies from the UGC catalogue (Nilson 1973) in respect to the neighbouring large-scale structures and found that they are aligned in the scale at least up to $2^{o}$, which corresponds to the linear scale $\sim 2 \times h^{-1} M p c$. Next Muriel and Lambas (1992), taking into account the $3 D$ distribution of galaxies obtained from their redshifts, determined that the spiral galaxies are oriented towards their nearest neighbours, while the elliptical galaxies are aligned on the scale smaller than $3 h^{-1} M p c$. Also Cabanela and Aldering (1998) noted that the anisotropy they found in the distribution of galaxies belonging to the Perseus Supercluster can be of local, and not global (for the entire supercluster) character.

The basic mechanism to generate galaxy rotation in the scenario of hierarchical clustering are the mutual tidal torque interactions (Wesson (1982) and White (1984) - basing on the ideas of Hoyle (1951)). In this scenario the distribution of spin angular momenta of galaxies should be random, but it was shown that the local tidal shear tensor can cause a local alignment of rotational axes (Dubinski 1992, Catelan and Theuns 1996, Lee and Pen 2000, 2001, 2002, Navarro, Abadi and Steinmetz 2004). Along these lines - as consistent with the tidal torque mechanism - were also reinterpreted the earlier results to indicate the alignment of galaxies (e.g. those of Flin and Godłowski 1986). On the other hand, some researchers, as e.g. Brook et al. (2010), still advocate the misalignment of angular momenta that occurs during hierarchical structure formation.

Brown et al. (2002) studying ellipticity of galaxies from the Supercosmos survey (mean redshift $z=0.1$ ) found that they are correlated in the scale between 1 and 100 arc min. The meaning of this observation is not clear, however the authors interpret it as a local effect, consistent with the theory of tidal interactions. Lee and Pen (2002) attempted to measure intrinsic alignment of galaxies out of observational data by the way of reconstructing the tidal shear tensor. However they did find the spin-shear correlation, their results are of doubtful value, due to a high noise level (Lee 2004). Thus the intrinsic correlations between galaxy spins and intermediate principal axes of the tidal shears were confirmed only recently Lee and Erdogdu (2007) and Lee (2010). Navarro, Abadi and Steinmetz (2004), analysing a sample of near spiral galaxies seen edge-on, found that their planes are perpendicular to the main plane of LSC. Lee (2004), who interpreted this fact as consistent with the tidal interactions model, basing on this scenario investigated analytically its consequences for the large-scale orientation of galaxies and found that his predictions agree with the distribution of orientations of nearby spiral galaxies. Trujillo, Carretero and Patiri (2006) determined, by probing the distribution of angles between rotational 
axes of galaxies and the vector connecting a given galaxy with the centre of a local void, that the spins of spiral galaxies located on the shells of the largest cosmic voids, are perpendicular to the direction towards its centre (i.e. along the direction of the envelope defined by the surrounding matter). Noh and Lee (2006a, 2006b), analysing nearby galaxies from Tully's catalogue (Tully 2000), found alignment of spiral galaxy planes perpendicular to the plane of a local pancake, which they interpret along the lines of the recently proposed scenario of broken hierarchy (Bower et al. 2005). In this scenario, instead of hierarchical clustering on all mass scales, there is an anti-hierarchical clustering on a small scale, since gravitational tidal effects give rise to objects of Zeldovich's pancake type (Zeldovich 1970) rather than objects of spherically collapsing halo. However, contrary to the classic pancake scenario, this effect occurs just locally on a small scale.

Hernandez et al. 2007 using sample of 11597 SDSS galaxies, present formulas to derive an estimate of the halo spin parameters $\lambda$ for any real galaxy, in terms of common observational parameters. For spirals they found a good correlation between empirical values of $\lambda$ and visually assigned Hubble types, showing usefulness of this physical parameter as one of classification tools.

Recently, there have been some attempts to investigate galaxy angular momenta on a large scale. Paz, Stasyszyn and Padilla (2008) analysing galaxies from the Sloan Digital Sky Survey catalogue found that the galaxy angular momenta are aligned perpendicularly to the planes of large-scale structures, while there is no such effect for the low-mass structures. They interpret this as consistent with their simulations based on the mechanism of tidal interactions. Jones, van der Waygaert and Aragon-Calvo (2010) found that the spins of spiral galaxies located within cosmic web filaments tend to be aligned along the larger axis of the filament, which they interpreted as "fossil" evidence indicating that the action of large scale tidal torques effected the alignments of galaxies located in cosmic filaments.

The largest scale alignment was found in the series of paper by Hutsemekers (Hutsemekers 1998, Hutsemekers and Lamy 2001, Hutsemekers et al. 2005) during analyzis of the alignment of quasar polarization vectors. In the paper Hutsemekers et al. (2005), based on a new sample of 355 quasars with significant optical polarization they fount that quasar polarization vectors are not randomly oriented over the sky. The polarization vectors appear coherently oriented or aligned over huge $(\sim$ $1 \mathrm{Gpc}$ ) regions of the sky. Furthermore, the mean polarization angle $\theta$ appears to rotate with redshift at the rate of $\sim 30^{\circ}$ per Gpc. While interpretations like a global rotation of the Universe can potentially explain the effect, the properties they observed qualitatively correspond to the dichroism and birefringence predicted by photon-pseudoscalar oscillation within a magnetic field. These results usually are not questioned, (with exeptions of Joshi et al. 2007 which not found any effects during analyzis of theirs sample (Jackson et al. 2007)), however the origin of this effect is still discussed. Possible interpretations of the alignment effect have been discussed in Hutsemekers (1998), Hutsemekers and Lamy (2001), Hutsemekers et al. (2005) and more recently by several authors (Jain et al. 2002, 2004, Ralston and 
Jain 2004, Hutsemekers et al. 2010, Poltis and Stojkovic 2010, Silantev et al 2010, Antoniou and Perivolaropoulos 2010, Agarwal, Kamal and Jain 2011).

Recognizing intrinsic alignment of galaxy spins is also important with regard to investigations of weak gravitational lensing, in which, according to Heavens, Refregier and Heymans (2000), the intrinsic spin alignment in galaxy pairs has to be taken into account, since otherwise it would affect the results in a systematic way. Crittenden et al. (2001) proved that at least in the scenario of tidal interactions, the effects of alignment can be distinguished from the effects of weak gravitational lensing, while Heymans et al. (2004) provided a detailed analysis of some method to determine intrinsic spin orientation in galaxy pairs as well as to remove them from the weak gravitational lensing considerations. From our point of view, it is of importance that they thus confirmed the presence of spin alignment in pairs of galaxies. Systematic effects occurring in weak gravitational lensing investigations were also discussed by Mandelbaum et al. (2005).

This brief review of results of studies of galaxy orientations indicates that there are no deciding arguments weighing in favour of any galaxy formation scenario to date. Most of them can be interpreted as supporting either the pancake scenario or the scenario of tidal interactions. One should note the fact that while in the pancake scenario involves an explicit mechanism to generate non-random orientations of galaxy rotational axes, there is no obvious mechanism of this kind in the other scenarios. Then it is necessary to more accurately recognize galaxy orientations within the structures of galaxies in order to look for further evidence supporting one of the scenarios. Such evidence could be provided by investigating orientation of galaxies within clusters.

\section{Results of investigation of galaxy orientations in clusters of galaxies}

The investigations of galaxy orientations in clusters have also quite a long history. Thompson (1976) found alignment of galaxy orientations in the Virgo and A2197 clusters. Adams (1980) discovered a bimodal distribution of galaxy orientations examining the combined data for seven galaxy clusters (A76, A179, A194, A195, A999, A1016, A2197). The orientation of principal axes of the clusters corresponded with one of those maxima. Helou and Salpeter (1982), studying 20 galaxies belonging to the Virgo cluster, found that their spins are not directed in random, however the nature of this non-random distribution was not too clear. Mac Gillivray and Dodd (1985a) investigated the distribution of orientation of galaxies in the Virgo cluster and showed that the galaxy planes are perpendicular to "the direction towards the cluster's centre", i.e. the galaxies' rotational axes are aligned towards that centre. Gregory, Thompson and Tifft (1981) found in the Perseus supercluster (A426, A262) and the groups around the galaxies NGC383 and NGC507 a bimodal distribution of galaxy position angles with two maxima. The position of one of them corresponds to the position angle of the Perseus supercluster. Here we have two populations of 
galaxies with almost perpendicular axes.

On the other hand, Bukhari (1998), as well as Bukhari and Cram (2003) studying orientation of galaxies within clusters did not recognize any alignment. Han, Gould and Sackett (1995) probed a region of the Local Supercluster with an enhanced density galaxies containing 94 objects. Analyzing a sample of 60 galaxies for which accurate values of spins were available, they found no alignment. Hoffman et al. (1989) for a sample of 85 galaxies with well-known spins from the Virgo cluster did not find any alignment either. Flin and Olowin (1991), Trevese, Cirimele and Flin (1992), Kim (2001), investigating isolated Abell clusters, detected just rudimentary traces of alignment. The similar results were obtained by Torlina, De Propris and West (2007) from studies of the Coma cluster and its vicinity. Gonzalez and Teodoro (2010) interpreted the alignment of just the brightest galaxies within a cluster as an effect of action of gravitational tidal forces. Correlation between the orientation of the brightest galaxy within a cluster and the cluster's large axis was also found by Sastry (1968), Carter and Metcalfe (1980), Binggeli (1982), Struble and Peebles (1985), Rhee and Katgert (1987), West $(1989,1994)$, van Kampen and Rhee (1990), Plionis (1994), Fuller et al. (1999) and Kim et al. (2002).

Godłowski and Ostrowski (1999) analysed in various coordinate systems orientation of galaxies in 18 Tully's groups within the Local Supercluster taken from Tully's catalogue (1988). As the fundamental coordinate system, the second, supergalactic coordinate system was assumed (Flin and Godłowski 1986) and the position of the coordinate system pole was varied (by $5^{\circ}$ at a time), both in supergalactic latitude $\mathrm{B}$ and in longitude $\mathrm{L}$. If the galaxy rotational axes preferably aligned in some plane, there would be a deficit of rotational axes oriented in the direction perpendicular to that plane, whereas if the orientations of galaxy rotational axes preferred some particular direction, then there should be an excess of galaxies with spins oriented along this direction. A strong correlation was found between those maxima and the line of sight towards the cluster, while there were no such correlations for the other analysed directions. The obtained excess of galaxy rotational axes oriented along the line of sight towards the cluster can suggest that we are dealing here with a systematic effect related to the process of deprojection of axes of galaxies from their optical images. The essential result of Godłowski and Ostrowski (1999) consists in discerning within the catalogue data a strong effect (further called the line-of-sight (LOS) effect) related to the process of deprojecting axes of galaxies from their optical images. It was shown that the very process of deprojection of galaxies off the catalogue data generates considerable systematic errors, which have to be taken into account in investigating galaxy orientations. The discerned effect mostly obscures any possible weak effects of galaxy orientation alignment in the examined clusters. The problem with deprojection of optical images had been earlier signalled by other authors, particularly Kapranidis and Sullivan (1983). In the work mentioned above (Godłowski and Ostrowski 1999) it was proved that the effect is significant for Tully's catalogue (1988). The main reason behind this is that in calculating galaxy inclination angles Tully assumed that the "true" ratio of axes 
of galaxies is 0.2 , which is a rather poor approximation, especially for non-spiral galaxies (Godłowski 2011).

This result presented above, pertains to the analysis basing on Tully's catalogue (1988), showing that in the data there is an excess of galaxies seen face-on. It should be stressed that by modelling the LOS effect for a sample galaxies taken off the UGC (Nilson 1973) and ESO (Lauberts 1982) catalogues and including the effects of true ellipticity (after Heidmann, Heidmann and de Vaucouleurs (1971)) and Holmberg (after Fouque and Paturel (1985)) it was found that the LOS effect, if any, is much smaller (Godłowski and Ostrowski 1999, Godłowski 2011). This is consistent with the result of Bahcall, Guhathakurt and Schneider (1990), who stated that there is no excess of elliptic galaxies seen face-on in the UGC, where is actually an excess of spiral galaxies seen edge-on, which can be accounted for either by the Holmberg effect or by "dropping" weak spiral galaxies, classified as stars, off the catalogue. To summarize, the results show that in examining galaxy orientations using their ellipticity, one has to make a very careful analysis of the available observational data, especially in regard of the Holberg and true ellipticity effects, as well as of possible occurrence of other systematic observational effects.

Problem of "true" shape of galaxies is also very important in the context of Tully Fisher (Tully and Fisher 1977) and Baryonic Tully Fisher relation (Freeman 1999, Mc Gaugh et al. 2000 and Gurovich et al. 2010 for the latest review). One of important problems for these relations is correction to edge-on inclination. The inclination angles are obtained by assuming that the "true" ratio of axes of galaxies is 0.2 , which, as it was noted above, is a rather poor approximation. The Tully Fisher relation is an important point where observations and the theory of galaxy angular momentum meet.

Orientation of galaxies in Tully's groups was examined again in Godłowski, Szydłowski and Flin (2005). In conclusion, it can be stated that no significant evidence was found to demonstrate alignment of galaxy rotation axes in the analysed Tully's groups. Also Aryal and Saurer (2005c), who investigated three Abell clusters of richness class zero S0794, S0797, and S0805, found that the galaxies in those poor cluster are oriented at random.

Godłowski, Baier and MacGillivray (1998) inspected galaxy orientations within the cluster Abell 754 basing on COSMOS/UKST Southern Sky Objects Catalogue (Yentis et al. 1991). They analysed the distributions of galaxy position angles as well as polar and azimuth angles, confirming the LOS effect recognized by Godłowski and Ostrowski (1999), which generates significant systematic errors in the process of deprojection. For that reason, the conclusions concerning galaxy orientations within the clusters under examination were based on the analysis of galaxy position angles. In this work, it was demonstrated that the distribution of galaxy orientations in the double cluster Abell 754 is non-random, with the galaxy planes perpendicular to the main plane of the cluster. The same method was applied to the cluster Abell 14 (Baier, Godłowski and MacGillivray 2003), where a non-random distribution of galaxy orientation was found too, but the direction of this alignment (in respect to 
the cluster's main plane) is perpendicular to that in Abell 754. According to Di Fazio and Flin (1988), such a discrepancy in preferred directions of galaxy orientation in respect to the main plane of the cluster can be related to existing two types of galaxy clusters: oblate and prolate ones.

While examining galaxy orientation in the clusters' substructures, it was found that for Abel 754 and Abel 14 clusters, the galaxies within their substructures are oriented in a non-random way, consistent with the orientation of galaxies within the entire cluster. A higher-mode analysis of the Fourier test (40) shows that the position angle distribution in those clusters can have even more sophisticated, bimodal character, probably related to the presence of substructures. However, since the maxima in the distribution for the entire cluster and its principal substructure are the same, this latter result should be regarded with caution.

It was confirmed that there is an effect recognized by Godłowski and Ostrowski (1999), giving rise to considerable systematic errors in the process of galaxy deprojection from the catalogue data. As this confirmation is based on the independent observational data, it means that the effect occurs not only for the data from Tully's catalogue (1988). Thus it could contribute to distortion of some of the earlier results concerning galaxy orientation. The main reason that the effect occurs with the COSMOS/UKST Southern Sky Objects Catalogue (Yentis et al. 1991) data, is apparently the fact that the catalogue does not provide morphological types of galaxies, making us to assume for all the galaxies that the "true" galaxy axis ratio $q_{0}=0.2$, which is, as it was already mentioned, a rather poor approximation, especially for non-spiral galaxies. However, the effect was found as much stronger than for Tully's groups in Godłowski and Ostrowski (1999). It may be due to the fact that COSMOS has difficulties with distinguishing between stars and galaxies (Bukhari and Cram 2003). In effect, the sample under examination can be contaminated by stars misclassified by COSMOS as galaxies. Furthermore, Cabanela and Aldering (1998) showed that some effect similar to the Holmberg effect can be considerable also when the data are taken off a catalogue in which galaxy sizes were measured through the method of digital scanning. Using the Minnesota Automap Plane Scanner (Pennington and al. 1993) they compiled the catalogue of galaxies belonging to the Pisces-Perseus supercluster, and basing on the analysis by Huizing (1994) noted that the external regions of spiral galaxies (in contrast to their internal regions) are optically thin, and thus the measured galaxy diameters should increase with their inclination, which in turn leads to their overrepresentation after cataloguing. However Cabanel and Aldering's analysis (1998) should be regarded with caution, it is evident the effect they found has to be taken into account in galaxy orientation investigations based on their ellipticity, when the data are taken off catalogues in which galaxy sizes are measured by digital scanning.

Summing up the results obtained by various authors, it can be stated that we have no satisfactory evidence to support the galaxy axis alignment in the groups and poor clusters of galaxies, while there is ample evidence of this kind for the rich 
clusters of galaxies. However, it should be emphasized that even where actually is alignment, there is no common preferred direction of galaxy spin orientations, and thus while in some clusters the direction perpendicular to the main plane of the cluster is preferred, in other cluster the galaxy spins tend to be oriented parallelly to the structure's main plane. The cited above results are not consistent with any of the three main scenarios of galaxy formation.

\section{Angular momenta of galaxies - observational aspects of Li's model}

If we now assume validity of Li's model, then from the analysis of observational data on orientation of galaxies it can be supposed that the structures with a mass typical for galaxy groups are just the structures with minimal angular momentum. For this reason, the orientation of galaxies in 18 Tully's groups was examined once again in Godłowski, Szydłowski and Flin (2005) with respect to distribution of angles: polar, azimuth and position ones in the supergalactic coordinate system for galaxies belonging to those clusters, using three statistical tests $\left(\chi^{2}\right.$, Fourier, and autocorrelation). The inspection of polar and azimuth angle distributions indicate possible occurring of rotation axis alignment in some of the analysed Tully's groups (groups 11, 31, 41, 51, 52). One should however have in mind that in the catalogue data (Tully 1988) there is a strong effect, recognized by Godłowski and Ostrowski (1999), related to the process of deprojection of axes of galaxies from their optical images. Therefore the distributions of supergalactic position angles were analysed independently. The results obtained from the analysis of position angles do not confirm the hypothesis of galaxy plane orientation alignment. In effect, it can be asserted that no sufficient evidence was found to support such an alignment in Tully's groups under examination. It should be stressed that the most significant effect was discovered in the most massive structure - Tully's group no. 11 (the Virgo cluster).

In the cited work (Godłowski, Szydłowski and Flin 2005), the authors considered also the relation between the angular momentum and the mass of a structure as based on the observational data. It was investigated how this image changes in dependence on the mass of structures made up of galaxies, beginning from the simplest ones, i.e. pairs of galaxies. These investigations showed that their angular momentum originates mainly from the orbital motion of galaxies (Karachentsev and Mineva 1984a, 1984b, Mineva 1987). Helou (1984), examining a sample of 31 galaxy pairs, found that an "anti-alignment" of spins of these galaxies. Parnovsky, Kudrya and Karachentsev (1997) recognized a weak alignment in physical pairs of galaxies. Alignment in pairs of spiral galaxies was also discerned by Pestana and Cabrera (2004). Intrinsic spin alignment in pairs of galaxies was independently confirmed by Heymans et al. (2004) within their research on weak gravitational lensing, where it was necessary to estimate and remove the effects related to alignment of galaxy orientations. Also the analysis of positions of the Milky Way's companions shows their 
non-random distribution (they are located perpendicularly to the Milky Way's disc), which can be regarded as their orbital alignment. Galaxies within compact groups rotate on prolate orbits about the group's centre (Tovmassian et al. 2001), which contributes to the system's total angular momentum. Thus it can be maintained that structures as galaxies and their companions, pairs of galaxies and compact groups of galaxies have a non-zero net angular momentum.

The more massive structures are groups and clusters of galaxies, for which there is no evidence of rotation. Moreover, Hwang and Lee (2007) examined dispersions and velocity gradient of 899 Abell clusters and found a possible rotation in only 6 of them. Any non-zero angular momentum of groups and clusters of galaxies would just come from possible alignment of galaxy spins. There is no sufficient evidence to support galaxy rotational axis alignment in groups and poor clusters of galaxies. Additionally, it is obvious that in the isolated Abell groups just the brightest galaxies manifest a rudimentary alignment (Flin and Olowin 1991, Trevese, Cirimele and Flin 1992, Kim 2001), while in the most numerous clusters as A754 (Godłowski, Baier and MacGilivray 1998), A14 (Baier, Godłowski and MacGilivray 2003), A1656 (Djorgovski 1983, Wu et al. 1998, Kitzbichler and Saurer 2003), a non-random galaxy orientation alignment was found. The most recent analysis of alignment in the Coma cluster was carried out by Adami et al. (2009), who concluded that alignment occurs only in some regions of the cluster and the galaxies of early and late spectral types have different orientations. Similarly, the preferred orientation is different in various parts of the cluster. The alignment of galaxy planes was recently found in the cluster 1689 (Hung and in 2010). This result is of importance as it has been the most remote cluster to have been looked for the alignment effect to date. Presence of non-random galaxy spin orientation has been ascertained both in the Local Supercluster and other superclusters, as the Hercules supercluster (Flin 1994), Coma/A 1367 (Djorgovski 1983, Garrido et al. 1993, Wu et al. 1997, Flin 2001) and the Perseus supercluster (Gregory, Thompson and Tift 1981, Flin 1988, 1989, Flin and Godłowski 1989b, Cabanela and Aldering 1998).

In Godłowski, Szydłowski and Flin (2005) the following observational situation was depicted. For such galaxy structures as pairs of galaxies and their compact groups, there is some evidence to indicate a non-zero angular momentum of the entire structure, while for the more massive structures, groups and poor clusters of galaxies evidence of this kind is absent. From examining rich galaxy clusters and superclusters the results were obtained, which showed a non-random orientation of galaxies within these structures, imparting a non-zero net angular momentum. Such dependence of a structure's angular momentum on its mass is consistent with the conclusions from Li's model.

This overall image was supported in subsequent studies into the topic of galaxy orientations. For instance, Yang et al. (2006) found, while Sales and Lambas (2009) and Wang et al. $(2009,2010)$ confirmed it, that the companions of central red galaxies are aligned along their large axes. Aryal and Saurer in a series of articles (Aryal 
and Saurer 2004, 2005b, 2005c, 2006, Aryal, Pudel and Saurer 2007) dealt with orientation of galaxies in 32 clusters of various numerousness, confirming (Aryal, Pudel and Saurer 2007) the situation that we do not find any galaxy orientation alignment in sparsely populated clusters, while such alignment is observed in a number of rich clusters of galaxies. However, either in Godłowski, Szydłowski and Flin (2005) or in Aryal, Pudel and Saurer (2007) only a qualitative and not quantitative analysis on this issue was provided.

Therefore Godłowski et al. (2010) examined orientation of galaxies in clusters both qualitatively and quantitatively and found a sharp increase of galaxy orientation alignment with numerousness of the cluster. The analysis was carried out on a sample of 247 Abell clusters with at least 100 objects each, taken off the Panko and Flin catalogue (2006), which had been based on the Muenster Red Sky catalogue (MRSS), covering the area of 5000 square degrees on the southern sky and providing data on 5.5 million galaxies. The catalogue is statistically complete to the value of $m=18.3 \mathrm{mag}$. The PF catalogue includes structures with at least 10 members with luminosities within the range from $\mathrm{m} 3$ through $m_{3}+3$, where $\mathrm{m} 3$ is the value of luminosity of the third brightest galaxy within a given region. The clusters were selected using the tessellation method of Voronoi (Panko, Juszczyk and Flin 2009).

For each cluster analysed in Godłowski et al. (2010), the distributions of position angles as well as of polar and azimuth angles were determined, both in the equatorial and in the supergalactic coordinate system. In analysis of these angle distributions the statistics of $\chi^{2}$ and Fourier tests were applied (Halley and Peebles 1975, Flin and Godłowski 1986, Godłowski 1993, 1994). The values of analysed statistics were found to increase sharply with the numerousness of a given cluster, while this effect was stronger for the polar and azimuth angles than for the position ones, and still stronger after limiting to galaxies more luminous than $m_{3}+3$, which proves that the effect is actually related to the clusters under examination.

In Godłowski et al. (2010) it was found that, contrary to the suggestions of Aryal and Saurer (2004, 2005b, 2005c, 2006), Aryal, Pudel and Saurer (2007), the orientation alignment of galaxies is weakly correlated with their morphological types according to the classification of Bautz-Morgan (BM), while the suggested by Plionis et al. (2003) correlation between the degree of galaxy orientation alignment and the dispersion of galaxy velocities in clusters was not confirmed.

However the results of Aryal and Saurer (2006) as well as those of Godłowski et al. (2010) are consistent the predictions of Li's model, they may be as well interpreted as an effect of tidal forces, according to the scenario of Catelan and Theuns (1996). The analyses of Noh and Lee (2006a, 2006b) also suggest that the linear theory of tidal interactions there should involve a relation between the galaxy orientation alignment and the structures' masses.

It should be emphasized that Li's model is obviously disputable and cannot provide explanation for all the observed effects, but it is still of interest as it accounts for the dependence of structures' angular momentum on their masses, which the 
other models have been not able to explain so far. However please note that the simple relation between the mass of a cosmic structure and its angular momentum expressed by the empirical relation $J \sim M^{5 / 3}$ is also predicted by Tidal Torque scenario.

\section{Summary}

One of the key issues of modern extragalactic astronomy is the problem of origin of galaxies. This work describes in detail various hypotheses of galaxy formation and discusses their connection to galaxy orientation. There are several scenarios of galaxy formation, which can explain a number of theoretical problems and observational results. However, none of them does account for all the difficulties concerning the process to form galaxies and their structures. The purpose of this work is to present the current observational status of galaxy orientation, and subsequently to check to what extent the existing models of formation of galaxies and their structures are consistent with observations. It also describes the theoretical and observational aspects of dependence of the structures' angular momentum on their mass and comprehensively discusses the results of investigations of galaxy orientation within various structures, finding that Li's theoretical model, in which galaxies form in a rotating universe, is supported by the observational evidence. An overview of possibilities and theoretical difficulties in constructing cosmological models involving rotation as well as of observational constraints on the actual amount of rotation of the Universe is also provided.

Studying orientation of galaxies is one of the most effective methods of testing the galaxy formation scenarios. In this paper the observational results of the investigations of angular momentum of galaxies was discussed and compared with the predictions of three classical scenarios of galaxy formations i.e. primordial turbulences, Zeldovich's pancake model and hierarchical clustering with mutual tidal torque interactions mechanism. The results of early investigations of alignment of rotational axes within cosmic structures were usually interpreted as consistent with Zeldovich's pancake model or the model of tidal torque interactions in the scenario of hierarchic clustering. Recently, some variants of tidal torque model, usually with hierarchical clustering scenario, have become commonly accepted. Only the pancake model involved some obvious mechanism of acquiring angular momentum by galaxies. Admittedly, in the most recent model of broken hierarchy the tidal effects also give rise to object of the kind of Zeldovich's pancakes (but on a small scale), however all the main models of formation of galaxies and their structures in the Universe encountered difficulties in accounting for the observed dependence of the structures' angular momentum on their masses. Tidal Torque scenario predict that the relation between the mass of a cosmic structure and its angular momentum is expressed by the empirical relation $J \sim M^{5 / 3}$. In Li's model, in which galaxies form in a rotating universe, there is a quite evident mechanism to impart the galaxies with angular momentum. Moreover, it provides for an attempt to relate the angular 
momentum of a structure to its mass. If only for these reasons, the model is of interest, while naturally it has its weak points. Its essential drawback consists in the fact that the observed amount of rotation of spiral galaxies cannot arise from the Universe's rotation alone, since the required amount of rotation of the Universe is too large in comparison with the detected anisotropies of cosmic background radiation.

The work reveals that the presence of non-zero angular momentum is contingent upon the structure's size. The presented observational situation is that the galaxies, their pairs and compact groups have a non-vanishing angular momentum. In the structures of mass corresponding to groups of galaxies this feature is not to be found, while in the clusters and superclusters alignment of galaxy orientation has been discerned, which indicates a non-zero net angular momentum. It was shown that such an observational depiction of dependence of angular momentum on the structure's size is consistent with Li's model.

Li's model assumes the global rotation of the Universe. Unfortunately, there is no relatively simple model of rotating universe derived from the general relativity theory, involving observables that could be used for its empirical testing. However, we can make some estimates of global rotation of the Universe on the grounds of the Newtonian equivalent of general relativity, while being fully aware that the Newtonian cosmology does not allow for describing actual evolution of the Universe, being but its conveniently applicable approximation. The situation is somewhat complicated by the fact that observationally it is very hard to distinguish the effects of global rotation from other effects, as the Casimir effect or dark radiation in the brane scenario of Randall-Sundrum. One can still estimate from the observational data the net contribution of all effects of this kind. It is shown that if it has a non-zero value, then one can explain the discrepancies between the values of matter density $\Omega_{m, 0}$ and of the Hubble constant derived from measurements of SNIa and CMBR. It allows also to account for the discrepancies between the observed and theoretical abundances of helium- 4 and deuterium.

The main assets of this work are:

(1) It was recognized that the presence of a negative term scaling as $(1+z)^{4}$ in the Friedmann equation (or its Newtonian equivalent) can be of various origin. Among the possible causes could be the cosmological model with global rotation, the FRW model in the brane scenario of Randall-Sundrum with dark radiation and the Casimir effect. Due to this, it is virtually impossible to separate the effects of rotation using the standard astronomical tests.

(2) Using the observational data concerning the Ia-type supernovae, radio galaxies, cosmic background radiation and baryon oscillations, a constraint on the net contribution of density parameters of fluids of negative density scaling as $(1+z)^{4}$ was determined.

(3) It was shown that the presence of such fluids of negative density allows for obtaining a flat model of the Universe with $\Omega_{m, 0}=0.3$, consistent both with the Ia-type supernovae and CMBR observations. 
(4) It was demonstrated that analysing the first peak in the CMBR power spectrum one gets a much stricter constraint on the sum of density parameters of negative fluids scaling as radiation than one derived earlier from the nucleosynthesis analysis.

(5) Observational results are presented to indicate that the galaxy orientation alignment is contingent upon the mass of a given structure: for Tully's groups of galaxies no satisfactory evidence was found for galaxy plane alignment, while there are non-random orientations of planes of galaxies belonging to the Abell clusters.

(6) The observational situation was depicted as follows: pairs and compact groups of galaxies reveal a non-vanishing angular momentum; for groups of galaxies there is no evidence for a non-zero angular momentum, while such evidence can be provided for clusters and superclusters of galaxies.

(7) It was shown that the galaxy orientation alignment increases with the numerousness of clusters, while it is weakly correlated with their morphological type according to the Baitz-Morgan (BM) classification.

(8) It was shown that Li's model, in which galaxies form in a rotating universe, predicts dependence of angular momentum on the structures' mass, which is consistent with the observational circumstances mentioned above.

(9) The deprojection effect was discussed, which can essentially modify the results of some earlier studies concerning galaxy orientation.

It should be explicitly stressed that none of the available scenarios of formation of galaxies and their structures in the Universe provides a complete explanation for all the observed properties of the object under consideration. In particular, this is the case with observations of galaxy orientation within structures of galaxies, while various models can account for various aspects of the galaxy formation process on different scales, as well as for various observational features of structures.

\section{Acknowledgments}

The author thanks prof. Piotr Flin for discussion. The author thanks the anonymous referee for suggestions and comments improving the paper.

\section{References}

\section{References}

1. Abbe, C., 1875 Amer. J. Sci. and Arts 9, 42

2. Abramyan, M. G., Sedrakyan, D.M., 1985 Astrophysics 23, 396

3. Adami, C., et al., 2009 Astron. Astroph. 493, 399

4. Adams, M.T., Strom, K.M., Strom, S.E., 1980 Astrophys. J. 238, 445

5. Agarwal, N., Kamal, A., Jain, P., 2011 Phys. Rev. D. 83, 065014

6. Akarsu, O., Kilinc, C. B., 2010 Gen. Rel. Grav. 42, 119

7. Antoniou, I., Perivolaropoulos, L. 2010 Journal of Cosmology and Astroparticle Physics, 12, 12 
8. Aryal, B., 2010 arXiv 1010.5585

9. Aryal, B., Kafle P.R., Saurer, W., 2008 Mon. Not. R. Astr. Soc. 389, 741

10. Aryal, B., Neupane, D., Saurer, W., 2008 Astroph and Space Science 314, 177

11. Aryal, B., Paudel, S., Saurer, W., 2007 Mon. Not. R. Astr. Soc. 379, 1011

12. Aryal, B., Paudel, S., Saurer, W., 2008 Astron. Astroph. 479, 397

13. Aryal, B., Saurer, W., 2000 Astron. Astroph. 364, L97

14. Aryal, B., Saurer, W., 2004 Astron. Astroph 425, 871

15. Aryal, B., Saurer, W., 2005a Astron. Astroph. 432, 431

16. Aryal, B., Saurer, W., 2005b Astron. Astroph. 432, 841

17. Aryal, B., Saurer, W., 2005c Mon. Not. R. Astr. Soc. 360, L25

18. Aryal, B., Saurer, W., 2006 Mon. Not. R. Astr. Soc.. 336, 438

19. Aryal, B., Kandel S.M., Saurer, W., 2006 Astron. Astroph. 458, 357

20. Astier, P., et al., 2006 Astron. Astrophys. 447, 31

21. Bahcall, J., Guhathakurta P., Schneider, D., 1990 Science, 248, 178

22. Baier, F., Godłowski, W., Mac Gillivray H.T., 2003 Astron. Astroph. 403, 847

23. Barrow, J.D., Juszkiewicz, R., Sonoda, D.H., 1985 Mon. Not. R. Astr. Soc. 213, 917

24. Bratek, Ł., Jałocha, J., Kutschera, M., 2007 Acta Phys. Polon. B. 38, 2513

25. Bietenholz M.F., Kronberg, P.P., 1984 Astrophys. J. 287, L1

26. Bietenholz, M.F., 1986 Astronom. J 91, 1249

27. Binggeli, B., 1982 Astron. Astrophys. 107, 338

28. Birch, P., 1982 Nature 298, 451

29. Birch, P., 1983 Nature 301, 736

30. Bower, R. G., et al. 2006 Mon. Not. R. Astr. Soc. 370, 645

31. Brook, C., et al., 2010, Astroph. J. 689, 678

32. Brosche, P,. 1986 Comm. Astroph. 11, 213

33. Brown, F.G., 1964 Mon. Not. R. Astr. Soc. 127, 517

34. Brown, F.G., 1968 Mon. Not. R. Astr. Soc. 138, 527

35. Brown, M.L., Taylor, A.N., Hambly, N.C., Dye, S., 2002 Mon. Not. R. Astr. Soc. 333,501

36. Bukhari, F.A., 1988 Astrophys. J. 333, 564

37. Bukhari, F.A., Cram, L.E., 2003 Astrop. and Space Science 283, 169

38. Bunn, E., Ferreira P., Silk, J., 1996 Phys. Rev. Lett. 77, 2883

39. Cabanela, J.E., Aldering, G., 1998 Astronom. J. 116, 1094

40. Cabanela, J.E., Dickey J.E., 1999 Astronom. J. 118, 46

41. Carrol, S.M., Field, G.B., 1997 Phys. Rev. Lett 79, 2394

42. Catelan, P., Theuns, T., 1996 Mon. Not. R. Astr. Soc.. 282436

43. Carrasco L., Roth, M. Serrano, A., 1982 Astron. Astroph. 106, 89

44. Carter, D. Metcalfe, J., 1980 Mon. Not. R. Astr. Soc. 191, 325

45. Chechin, L. M. 2010 Astron. Reports 54, 719

46. Ciufolini, I., Wheeler, J., 1995 Gravitation and Inertia Princeton University Press, Princeton

47. Collins, C.B., 1985 Journal of Math. Phys. 26 (8) 2009

48. Collins, C.B., Hawking, S.W., 1973 Mon. Not. R. Astr. Soc. 162, 307

49. Crittenden, R., Natarajan, P., Pen, U., Theune, T., 2001 Astrophys. J. 559, 552

50. Dai, W. S., Liu, R.L., Hu, F.X., 1978 Acta Astron. Sinica 1924

51. Daly, R., Djorgovski, S.G., 2003 Astrophys. J. 597, 9

52. Daly, R., Djorgovski, S.G., 2004 Astrophys. J. 612, 652

53. Danver, C.G., 1942 Annals of the Observatory of Lund 10, 7

54. Dekel, A., 1985 Astrophys. J. 298, 461

55. Demiański, M., Doroshkevich, A.G., 2007 Phys. Rev. D. 75, 13517 
56. Demiański, M., Griszczuk, L.P., 1972 Commun. Math. Phys. 25, 233

57. di Fazio, A., Flin, P., 1988 Astron. Astroph. 200, 5

58. Djorgovski S., 1983 Astrophys. J. 274, L11

59. Doroshkevich, A.G., 1970 Astrofizika 6, 581

60. Doroshkevich, A.G., 1973 Astroph. Lett. 14, 11

61. Doroshkevich, A.G., Saar, E.M., Shandarin, S.F., 1978 in: The Large Scale Structure of the Universe, IAU-Symp. No. 79, D. Reidel, Dordrecht, p.423

62. Dubinski, J., 1992 Astroph. J. 401, 441

63. Efstathiou, G., Bridle S.L., Lasenby, A.N., Hobson, N.P., Ellis, R.S., 1999 Mon. Not. R. Astr. Soc. 303 L47

64. Efstathiou, G.A., Silk, J., 1983 The Formation of Galaxies, Fundamentals of Cosm. Phys. 9, 1

65. Eisenstein, D.J., Bunn, E.F., 1997 Phys. Rev. Lett. 79, 1957

66. Eisenstein, D.J. et al., 2005 Astrophys. J. 633, 560

67. Ellis, G.F.R., 1966 Journal of Mathemat. Phys. 8, (5) ,1171

68. Ellis, G.F.R., 1973 in: Carges Lecture in Physics Vol. 6 ed. E. Schatzman, New York Gordon and Brach Science Publishers, p.1

69. Ellis, G.F.R., Olive, K. 1983 Nature 303, 679

70. Fil'chenkov, M.L., 2003 in Frontiers of Particle Physics ed. E. Studenikin, World Scientific Singapore, p. 284

71. Fil'chenkov, M.L., 2005, IJMPA 20,2388

72. Fliche, H.H., Souriau, J.M., 1990 Astron. Astroph. 233, 317

73. Flin, P.. 1988 Mon. Not. R. Astr. Soc. 235, 857

74. Flin,P., 1989 in: Morphological Cosmology, eds. P. Flin H.W. Duerbeck, Springer Verlag, Berlin, p.124

75. Flin, P., 1994 in: Cosmological Aspects of X-Ray Clusters of Galaxies ed. W.C. Seitter, Kluwer Academic Press, Dordrecht, p.363

76. Flin, P., 1995 Comments Astrophys., 18, 81

77. Flin, P., 2001 Mon. Not. R. Astr. Soc. 325, 49

78. Flin, P., Godłowski, W., 1986 Mon. Not. R. Astr. Soc. 222, 525

79. Flin, P., Godłowski, W., 1989a Sov. Astron. Lett. 15, 374 (Pisma w Astronomiczeskij Zurnal 15, 869)

80. Flin, P., Godłowski, W., 1989b in: ASLL Vol.155, Astronomy, Cosmology, and Fundamental Physics eds. M. Caffo, R. Fanti, G. G. Giacomelli, A. Renzini, Kluwer Academic Press, Dordrecht p.418

81. Flin, P., Godłowski, W., 1990 Sov. Astron. Lett. 65, 209 (Pisma w Astronomiczeskij Zurnal 16, 490)

82. Flin, P., Olowin, R.P., 1991 in: Physical Cosmology, eds. A.Blanchard, L. Celniker, M. Lachieze-Rey, Tran Thanh Van, Edition Frontiere, Gif-sur-Yvette, p.512

83. Fouque, P., Paturel, G., 1985 Astron. Astroph. 150, 192

84. Freedman et al., 2001, Astroph. J. 553, 47

85. Freeman, K., 1999 in: The Low Surface Brightness Universe, eds. J.I. Davies, C. Impey, S. Phillipps, San Francisco ASP Conf. Ser. Vol. 170, p.3

86. Fuller, T.M., West, M.J., Bridges, T.J., 1999 Astroph. J. 519, 22

87. Gamow, G., 1946 Nature 158, No 4016, 549

88. Gamow, G., 1952 Phys. Rev. 86, 251

89. Garrido J.L., Battaner, E., Sanchez-Saavedra, M.L., Florido, E., 1993 Asron. Astroph. 271,84

90. Goedel K., 1949 Rev. Mod. Phys 21, 447; 2000 GRG 32,1409

91. Godłowski, W., 1993 Mon. Not. R. Astr. Soc. 265, 874 
92. Godłowski, W., 1994 Mon. Not. R. Astr. Soc. 271, 19

93. Godłowski, W., 1995 Astro. Lett. and Comunications 31, 341

94. Godłowski, W., 2011 (in preparation)

95. Godłowski, W., Baier, F., Mac Gillivray, H.T., 1998 Astron. Astroph. 339, 709

96. Godłowski, W., Ostrowski, M., 1999 Mon. Not. R. Astr. Soc. 303, 50

97. Godłowski, W., Piwowarska, P., Panko, E., Flin, P., 2010 Astrophys. J. 723, 985

98. Godłowski, W., Szydłowski, M., Flin, P., Biernacka, M., 2003a Gen. Rel. Grav. 35, (5), 907

99. Godłowski, W., Szydłowski, M., Flin, P., 2005 Gen. Rel. Grav. 37, (3) 615

100. Godłowski, W., Szydłowski, M., 2003b Gen. Rel. Grav. 35, (12), 2171

101. Godłowski, W., Szydłowski, M., 2006 Phys. Lett. B. 642, 13

102. Gonzalez-Sanchez, A., Teodoro L., 2010 Mon. Not. R. Astr. Soc.404, L11

103. Gron, O., Soleng, H., 1987 Nature 328, 501

104. Gregory, S.A., Thompson, L.A., Tifft, W.G., 1981 Astrophys. J. 243, 411

105. Gurovich, S., Freeman, K., Jerjen, H., Puerari, I., 2010 Astronom. J. 140, 663

106. Han, C., Gould, A., Sackett, P., 1995 Astrophys. J. 445, 46

107. Hawking, S.W., 1969 Mon. Not. R. Astr. Soc. 142, 129

108. Hawley, D.L., Peebles, P.J.E., 1975 Astronom. J. 80, 477

109. Heavens, A., Peacock, J., 1988 Mon. Not. R. Astr. Soc. 232, 339

110. Heavens, A. Refregier, A. Heymans, C., 2000 Mon. Not. R. Astr. Soc. 232, 339

111. Heckmann, O. Schucking, E., 1959 Handbuch der Physik 53, 489

112. Heckmann, O., 1961 Astronom. J. 66, 599

113. Heidmann, J., Heidmann, N., de Vaucouleurs G.,1971 Mem. R. Astr. Soc. 75, 85

114. Helou, G., 1984 Astrophys. J. 284, 471

115. Helou, G., Salpeter, E.E., 1982 Astroph. J. 252, 75

116. Hernandez, X., Park, C., Cervantes-Sodi, B., Choi, Y., 2007 Mon. Not. R. Astr. Soc. 375,163

117. Heymans, C., et al., 2004 Mon. Not. R. Astr. Soc. 347, 895

118. Hofman, G.L., i.in., 1989 Astrophys. J. Supp. 69, 65

119. Holmberg, E., 1946 Medd. Lund. Astron. Obs. Ser. VI, Nr.117

120. Holmberg, E., 1958 Medd. Lund. Obs. II, 136

121. Holmberg, E., 1975 in: Galaxies and the Universe, eds. A.Sandage, M.Sandage, J.Kristian, University of Chicago Press, Chicago, p.123

122. Hoyle, F., 1951 in: Problems of Cosmological Areodynamics: Proceedings of a Symposium on Motion of Gaseous Masses of Cosmical Dimensions Paris 1949, eds. J.J.M. Burgeres, H.C. van de Hulst, p.195

123. Hu, F.X., Wu G.X., Su H.J., Liu, Y.Z., 1995 Astron. Astrop. 302, 45

124. Hu, F.X., Yuan Q.R., Su H.J., Wu G.X., Liu, Y.Z., 1998 Astrophys. J. 495, 179

125. Hu F.X., Wu G.X., Song G.X., et al., 2006 Astron. Space Sci 302, 42

126. Hung, L-W., Banados, E., De Propris, R., West, M. J. 2010, Astroph. J. 720, 1485

127. Hutsemekers, D. 1998 Astron. Astrophys. 332, 410

128. Hutsemekers, D., Lamy, H. 2001 Astron. Astrophys. 367, 381

129. Hutsemekers, D., Cabanac, R., Lamy, H., Sluse, D., 2005 Astron. Astrophys. 441, 915

130. Hutsemekers, D., Borguet, B., Sluse, D., Cabanac, R., Lamy, H. 2010 Astron. Astrophys. 520, L7

131. Hwang, H.S., Lee M.G., 2007 Astroph. J. 662, 236

132. Huizinga J.E., $1994 \mathrm{PhD}$ thesis Univ. Groningen, The Netherlands

133. Ichiki, K., et al., 2002 Phys. Rev. D 66, 043521.

134. Ichiki, K., et al., 2003 Phys. Rev. D 68, 083518. 
135. Iorio, L., 2010, Journal of Cosmology and Astroparticle Physics 8, 30

136. Jaaniste, J., Saar, E., 1977 Tartu Obs., Preprint A-2

137. Jaaniste,J., Saar,E., 1978 in: The large scale structures of the Universe., eds. M.S.Longair and J.Einasto, D.Reidel, Dordrecht (IAU Symp. 79), p.488

138. Jackson, N., Battye, R. A., Browne, I. W. A., Joshi, S., Muxlow, T. W. B., Wilkinson, P. N. 2007 Mon. Not. R. Astr. Soc. 376, 371

139. Jain, P., Ralston, J.P., 1999 Mod. Phys. Letters A14, 417

140. Jain, P., Panda, S., Sarala, S. 2002, Phys. Rev. D, 66, 085007

141. Jain, P., Narain, G., Sarala, S. 2004, Mon. Not. R. Astr. Soc. 347, 394

142. Jain, P., Modgil, M.S., Ralston, J.P., 2007 Mod. Phys. Lett. A 221153

143. Jones, B., van der Waygaert R., Aragon-Calvo M., 2010 Mon. Not. R. Astr. Soc. 408, 897

144. Joshi, S.A., Battye, R. A., Browne, I. W. A., Jackson, N., Muxlow, T. W. B. Wilkinson, P. N. 2007 Mon. Not. R. Astr. Soc. 380, 162

145. Kapranidis, S., Sullivan, W.T. III., 1983 Astron. Astrophys. 118, 33

146. Karachentsev, I.D., Mineva, V.A., 1984a Sov. Astr. Lett. 10, 105,

147. Karachentsev, I.D., Mineva, V.A., 1984b Sov. Astr. Lett. 10, 235,

148. Kashikawa, N., Okamura, S., 1992 PASJ 44, 493

149. Kendall, D.G., Young G.A., 1984 Mon. Not. R. Astr. Soc. 207, 637

150. King, A.R., Ellis G.F.R., 1973 Commun. Math. Phys. 31, 209

151. Kim, R., 2001 in: American Astronomical Society, 199 AAS Meeting, Bulletine of the American Astronomical Society vol 33, p.1521

152. Kim, R., et al., 2002 in: Tracing Cosmic Evolution with Galaxy Clusters, eds S. Borgani, M. Mezzetti, R. Valdarnini, San Francisco ASP Conf. Proc. Vol. 268, p.395

153. Kindl, A., 1987, Astronom. J. 93, 1024

154. Kitzbichler, M.G., Saurer, W., 2003 Astroph. J 590, L9

155. Kogut, A., Hinshaw, G., Banday, A., 1997 Phys. Rev. D 55, 1901

156. Krasiński A., 1975 J. Match Phys. 16, 125

157. Krasiński A., 1997 in: Homogeneous Cosmological Models Cambridge University Press Cambridge, p.317,

158. Krasiński, A., 1998a J. Match Phys. 39, 380

159. Krasiński, A., 1998b J. Match Phys. 39, 401

160. Krasiński, A., 1998c J. Match Phys. 39, 2148

161. Krasiński, A., 1999 The Ultimate extension on the Bainchi cassification for rotation dust models, in: "On Einstain's path, essays in honor of Engelbert Schucking ed. A.Harvey Springer New York, p.283

162. Krasiński, A., 2001a J. Match Phys. 42, 355

163. Krasiński, A., 2001b J. Match Phys. 42, 3628

164. Lambas, D., Groth, P., Peebles, P.J.E., 1988 Astronom. J. 95, 975

165. Lanczos, K., 1924 Z. Phys. 21, 73

166. Lauberts A., 1982 ESO/Uppsala Survey of the ESO B Atlas. ESO Garching

167. Lee, J., 2010 arXiv1011.5755

168. Lee, J., Erdogdu, P., 2007 Astrophys. J. 641, 1248

169. Lee, J., Penn, U., 2000 Astrophys. J. 532, L5

170. Lee, J., Penn, U., 2001 Astrophys. J. 555, 106

171. Lee, J., Penn, U., 2002 Astrophys. J.567, L111

172. Lee, J., 2004 Astrophys. J. 614, L1

173. Li, Li-Xin., 1998 Gen. Rel. Grav. 30, 497

174. Loredo, T.J., Flanganan, Wasserman, I.M., 1997 Phys. Rev. D 56, 7507

175. MacGillivray, H.T., Dodd, R.J., McNally, B.V., Corwin, Jr. H.G., 1982a Mon. Not. 
R. Astr. Soc. 198, 605

176. MacGillivray, H.T., Dodd, R.J., McNally, B.V., Lightfoot, J.F., Corwin, Jr.H.G., Heathcote, S.R., 1982b Astrop. Space Sci. 81, 231

177. MacGillivray, H.T., Dodd, R.J., 1985a in: ESO Workshop on the Virgo Cluster, ESO, ed. B. Binggeli Garching bei Munchen, p.217

178. MacGillivray, H.T., Dodd, R.J., 1985b, Astron. Astrophys. 145, 269

179. Mackrossan, M.N., 1987, Astr. And Space Science 133, 403

180. Mandelbaum et al., 2005 Mon. Not. R. Astr. Soc. 361, 1287

181. McGaugh, S.S., Schombert, J.M., Bothun, G.D., de Blok, W.J.G., 2000 Astrophys. J. 533, L99

182. Mineva, V.A., 1987, Sov. Astr. Lett. 13, 150

183. Molina-Paris, C., Visser, M., 1999 Phys. Lett. B. 455, 99

184. Muradian, R. M., 1975 Preprint JINR Dubna P2-8585

185. Muradian, R. M., 1980 Astrop. and Space Science 69,339

186. Muriel, H., Lambas D.G., 1992 Astron. J. 103, 393

187. Navarro, J.F., Abadi, M.G., Steinmetz M., 2004 Astrophys. J. 613, L41

188. Nilson,P., 1973 Uppsala General Catalogue of Galaxies (Uppsala Astr. Obs. Ann. V., vol.1)

189. Nilson,P., 1974 Uppsala Astr. Observatory Report No 3

190. Nodland, B., Ralston, J. P., 1997a Phys. Rev. Lett. 78, 3043

191. Nodland, B., Ralston, J. P., 1997b Phys. Rev. Lett. 79, 1958

192. Noh, Y. Lee, J., 2006a astro-ph /0602575

193. Noh, Y., Lee, J. 2006b Astrophys. Lett. 652, L71

194. Nordsiek, K.H., 1973 Astroph. J. 184, 735

195. Obukhov, Y., 2000 in: Cooloquium on Cosmic Rotation eds. M Scherfer, T. Chrobok, M.Shefaat Wissenschaft und Tecnik Verlag, Berlin, p.23

196. Obukhov ,Y., Chrobok T., Scherfner M. 2002 Phys. Rev. D. 66,043518

197. Oepik, X.,: 1970, Irish Astron. J. 9, 211

198. Ozernoy, L.M.: 1978, in: The Large Scale Structure of the Universe, IAU-Symp. No. 79 D.Reidel, Dordrecht, p.427

199. Panko, E., Flin, P. 2006 Journal Astron. Data, 12, 1

200. Panko, E., Juszczyk, T., Flin, P. 2009 Astron. J. 138, 1709

201. Parnovsky, S.L., Karachensew, I.D,. Karachentseva,V.E., 1994 Mon. Not. R. Astr. Soc. 268, 665

202. Parnovsky, S.L., Kudrya Y., Karachensew,I.D., 1997 Astronomy Letters 23, 504 (Pisma w Astronomiczeskij Zurnal 23, 576)

203. Paz, D.J, Stasyszyn, F., Padilla, N. D. 20081994 Mon. Not. R. Astr. Soc. 389, 1127

204. Peebles, P.J.E., 1969 Astrophys. J. 155, 393

205. Pennington, R., L., 1993 Pasp 105,521

206. Perlmutter S., et al., 1998 Nature 391, 51

207. Perlmutter S., et al., 1999 Astrophys. J. 517, 565

208. Pestana, J., Cabrera, J., 2004 Mon. Not. R. Ast. Soc. 353, 1197

209. Phinney, E.S., Webster, R.L., 1983 Nature 301, 735

210. Phinney, E.S., Webster, R.L, Kendall, D.G., Young, G.A. 1984 Mon. Not. R. Astr. Soc. 207, 637

211. Plionis, M., 1994 Astroph. J. Supp. 95, 401

212. Plionis, M., Benoist, C., Maurogordato, S., Ferreri, C., Basilakos, S., 2003, Astrophys. J. 594, 144

213. Poltis, R., Stojkovic, D. 2010 Phys. Rev. Lett. 105, 161301

214. Pontzen, A., Challinor, A., 2007, Mon. Not. R. Ast. Soc. 380, 1387 
215. Ralston, J.P., Jain, P., 2004 Int. J. Mod. Phys. D13, 1857

216. Randal, L., Sundrum, R., 1999 Phys. Rev. Lett. 83, 3370

217. Raychaudhuri, A.K., 1979 Theoretical Cosmology, Clarendon Press Oxford

218. Reinhardt, M., 1971 Astrophy. And Space Science 10, 363

219. Reinhardt, M., Roberts, M.S., 1972 Astrophys. Lett. 12, 201

220. Rhee, G., Katgert, P., 1987 Astron. Astrophys. 183, 217

221. Riess, A., et al., 1998 Astron. J. 116, 1009

222. Riess, A., i.in., 2004 Astrophys. J. 607, 665

223. Riess, A., i.in., 2007 Astrophys. J. 659, 98

224. Rothman, T., Ellis, G.R.F., 1986 Phys. Lett. B 180, 19

225. Sales, L., Lambas, D.G., 2009 Mon. Not. R. Astr. Soc. 395, 1184

226. Sastry, G.N., 1968 PASP 80, 252

227. Senovilla, J., Carlos, F. Sopuerta, C.F. Szekeres, P., 1998 Gen. Rel. Grav. 30, 389

228. Shandarin, S.F., 1974 Sov. Astr. 18, 392

229. Silk J., 1970 Mon. Not. R. Astr. Soc. 147, 13

230. Silantev, N. A., Gnedin, Y. N., Piotrovich, M. Y., Natsvlishvili, T. M., Buliga, S. D., 2010 Astrophysics, 53, 453

231. Sistero, R.F., 1983, Astroph. Lett., 23, 235

232. Sousa, A.A., Pereira R.B., Silva A.C., 2008, arXiv 0803.1481 (Gravitation and Cosmology 16, 25)

233. Spergel, D.N., et al., 2007 Astroph. J. Suppl. 170, 377

234. Steigman, G., Turner, M.S., 1983 Phys. Lett. B. 128, 295

235. Struble, M.F., Peebles, P.J.E., 1985 Astronom. J. 90, 582

236. Su, S.C., Chu, M.C., 2009 Astroph. J. 703, 354

237. Sunyaev, A.R. Zeldovich, Ya.B., 1972 Astroph. Sp. Sci. 7, 1

238. Szekeres, P., Rankin, R., 1977 Australian Math. Soc. B 20, 114

239. Szydłowski, M., Godłowski, W., 2005 Gen. Rel. Grav. 37, 907

240. Szydłowski, M., Godłowski, W., Krawiec, A., Golbiak, J., 2005 Phys. Rev. D 72, 063504

241. Thompson, L.A., 1976 Astrophys. J. 209, 22

242. Tippet B., Lake K., 2004 gr-qc/0409088

243. Torlina, L., De Propris, R., West, M.J., 2007 Astroph. J. 660, L97

244. Tovmassian, H.M., Chavushian, V., Martinez, O., Tiersch, H., Yam, O., 2001 in: Galaxies: The third dimension eds M.Rosado et al. ASP Conf. Ser 285, p.262

245. Trevese, D., Cirimele, G., Flin, P., 1992 Astronom. J. 104, 935

246. Trujillo, I. Carretero C., Patri G., 2006 Astroph. J. 640, L111

247. Tully, R.B., 1986 Astroph. J. 303, 25

248. Tully, R.B., 1988 Nearby Galaxy Catalogue., Cambridge University Press

249. Tully, R.B., 2000 Nearby Galaxy Catalogue., unpublished

250. Tully, R.B., Fisher, J.R., 1977 Astron. Astrophys. 54, 661

251. Turner, D.G., 2010a Astroph. And Space Science 326, 219

252. Turner, D.G., 2010b Odessa Astronomical Publications Vol 23 (in press)

253. Ungruhe, R., Saiter, W.C., Durbeck, H.W.., 2003, Journal Astronomical Data 9, 1

254. de Vaucouleurs, G., de Vaucouleurs, A., 1964 Reference Catalogue Bright Galaxies, University of Texas Monographs in Astronomy, University of Texas Press, Austin

255. Vishwakarma, R. G., 2001,Gen. rel. Grav. 331973

256. Vishwakarma, R. G., 2006, astro-ph/0404371 (Space Time and Substance 7, 53)

257. Vishwakarma, R.G., Singh, P., 2003 Clas. Quantum Grav. 202033

258. Wang, Y., et al. 2009 Astroph. J. 703, 951

259. Wang, Y., et al. 2009 Astroph. J. 718, 762 
260. Wang, Y., Tegmark, M., 2004 Phys. Rev. Lett. 92, 241302

261. van Kampen , E., Rhee G. 1990 Astron. Astroph. 237, 283

262. Wardle, J.F.C., Perley R.A., Cohen M.H., 1997 Phys. Rev. Lett. 79, 1801

263. Weinberg, S. 1972 Gravitation and Cosmology, Principles and Applications of the General Theory of Relativity, J.Wiley New York

264. von Weizsaeker, C.F., 1951 Astrophys. J. 114, 165

265. Wesson, P. S., 1979 Astron. Astrophys. 80, 296

266. Wesson, P. S., 1981 Phys. Rev. D. 23, 1730

267. Wesson, P. S., 1982 Vistas Astron. 26, 225

268. Wesson, P. S., 1983 Astron. Astrophys. 119, 313

269. West, M.J., 1989, Astrophys. J. 347, 610

270. West, M.J., 1994 Mon. Not. R. Astr. Soc. 268, 79

271. White, S.D.M., 1984 Astrophys. J. 286, 38

272. Wu G.X., Hu F.X., Su H.J., Liu Y.Z., 1997 Astron. Astrophys. 323, 317

273. Wu G.X., Hu F.X., Su H.J., Liu Y.Z., 1998 Chin. Astron. Astrophys. 22, 17

274. Wu G.X., 2006 Astron. Astrophys. 452, 807

275. Wyatt, S., Brown F.G., 1955 Astronom. J. 60, 415

276. Yang, X. et al., 2006 Mon. Not. R. Ast. Soc.369, 1293

277. Yentis D.J. et al., 1991 Digitised Optical Sky Surveys eds H.T. MacGillivray, E.B. Thompson, Dordrecht p.67

278. Zeldovich, B.Ya., 1970 Astron. Astroph. 5, 84

279. Zeldovich, B.Ya., 1978 in: The Large Scale structure of the Universe eds. M.S. Longair J.Einasto D.Reidel, Dordrecht, (IAU Symp. 79), p.409 